\title{
Article \\ Quantitative Analysis of 18 Marker Components in the Traditional Korean Medicine, Cheongsangbangpung-Tang, Using High-Performance Liquid Chromatography Combined with Photodiode Array Detector
}

\author{
Chang-Seob Seo *(D) and Hyeun-Kyoo Shin
}

Citation: Seo, C.; Shin, H. Quantitative Analysis of 18 Marker Components in the Traditional Korean Medicine, CheongsangbangpungTang, Using High-Performance Liquid Chromatography Combined with Photodiode Array Detector. Appl. Sci. 2021, 11, 14 . https://dx.doi.org/ 10.3390/app11010014

Received: 7 December 2020 Accepted: 19 December 2020

Published: 22 December 2020

Publisher's Note: MDPI stays neutral with regard to jurisdictional claims in published maps and institutional affiliations.

Copyright: (c) 2020 by the authors. Licensee MDPI, Basel, Switzerland. This article is an open access article distributed under the terms and conditions of the Creative Commons Attribution (CC BY) license (https: / / creativecommons.org/ licenses/by/4.0/).
Herbal Medicine Research Division, Korea Institute of Oriental Medicine, Daejeon 34054, Korea; hkshin@kiom.re.kr

* Correspondence: csseo0914@kiom.re.kr; Tel.: +80-42-868-9361

\begin{abstract}
Cheongsangbangpung-tang (CSBPT) is a traditional herbal medicine that has been used in many regions of Asia, including Korea, China, and Japan, for the treatment of purulent inflammation and eczema on the face. In this study, a method for the simultaneous analysis of 18 marker components, geniposide (1), coptisine chloride (2), prim-O-glucosylcimifugin (3), berberine chloride (4), liquiritin apioside (5), liquiritin (6), ferulic acid (7), narirutin (8), 5-O-methylvisammisoide (9), hesperidin (10), arctigenin (11), baicalin (12), oxypeucedanin hydrate (13), wogonoside (14), baicalein (15), arctiin (16), glycyrrhizin (17), and pulegone (18), was developed for quality control of CSBPT. The novel approach, which is based on high-performance liquid chromatography (HPLC) separation coupled with photodiode array detection, was verified by the assessment of linearity, limit of detection, limit of quantification, accuracy, recovery, and precision. Analysis of CSBPT by using the established assay revealed that compounds 1-18 were present in concentrations of $0.27-18.31 \mathrm{mg} / \mathrm{g}$.
\end{abstract}

Keywords: quantitative analysis; Cheongsangbangpung-tang; purulent inflammation; highperformance liquid chromatography

\section{Introduction}

Oriental medical prescriptions consisting of a combination of two or more medicinal herbs and containing many components have been widely used in Asian countries, such as Korea, China, and Japan, to enhance human health and prevent various diseases. However, there is a limit to continuous use without standardization of these herbal medicine prescriptions. Therefore, standardization for their continuous efficacy and quality control must be performed.

Cheongsangbangpung-tang (CSBPT; "Qing-Shang-Fang-Feng-Tang" in Chinese and "Seijo-bofu-to" in Japanese) is an oriental medical prescription that has been used in the treatment of purulent inflammation including acne and boils on the face and head [1,2]. CSBPT was first recorded in Gogeumuigam of Gong Shin in the Ming Dynasty of China, and it has also been included in Dongeuibogam of Heo Jun in the Joseon Dynasty of Korea [1,2]. This prescription consists of a combination of 12 medicinal herbs: Saposhnikoviae Radix, Angelicae Dahuricae Radix, Forsythiae Fructus, Platycodonis Radix, Scutellariae Radix, Cnidii Rhizoma, Schizonepetae Spica, Gardeniae Fructus, Coptidis Rhizoma, Aurantii Fructus Immaturus, Menthae Herba, and Glycyrrhizae Radix et Rhizoma, in 2.5:2.7:2.7:2.7:2.3:2.3:1.7:1.7:1.7:1.7:1.7:1.0 ratio [2].

Studies have been reported on the efficacy of CSBPT with respect to its antibacterial effects on Propionibacterium acnes and Staphylococcus aureus [3-5], its effect on atopic allergy [6], skin regeneration, wrinkle improvement, whitening, and moisturizing effects [1] Saruwatari et al. [7] assessed the safety of this prescription in a study on herbal drug interactions with cytochrome P450 (CYPs), N-acetyltransferase 2, and xanthine oxidase. 
Recently, Yang et al. [8] conducted and reported a pilot study on the effectiveness and safety of chronic urticarial in combination with Xiao-Feng-San and Qing-Shang-Fang-Feng-Tang.

The main ingredients of each medicinal herb that makes up CSBPT are as follows: chromones (e.g., cimifugin, prim-O-glucosylcimifugin, and 5-O-methylvisammioside) from Saposhnikoviae Radix [9], coumarins (e.g., imperatorin, phellopterin, and isoimperatorin) from Angelicae Dahuricae Radix [10], debenzylbutyrolactone lignans (e.g., matairesinol, arctiin, and arctigenin) from Forsythiae Fructus [11], platycosides (e.g., platycodon D and platycodin D2) from Platycodonis Radix [12], flavonoids (e.g., baicalin, wogonoside, baicalein, and wogonin) from Scutellariae Radix [13], phenylpropanoids (e.g., ferulic acid) and miscellaneous (e.g., z-ligustilide) from Cnidii Rhizoma [14], essential oils (e.g., pulegone) from Schizonepetae Spica [15], iridoid glycosides (e.g., geniposide) from Gardeniae Fructus [16], alkalids (e.g., berberine chloride and coptisine chloride) from Coptidis Rhizoma [17], flavonoids (e.g., narirutin, naringin, and hesperidin) from Aurantii Fructus Immaturus [18], essential oils (e.g., menthol and menthone) from Menthae Herba [19], and triterpenes (e.g., glycyrrhizin) and flavonoids (e.g., liquiritin apioside and liquiritin) from Glycyrrhizae Radix et Rhizoma [20].

There have been many simultaneous analysis methods reported for the quality assessment of each constituent herbal medicine of CSBPT based on high-performance liquid chromatography (HPLC), gas chromatography, and liquid chromatography-mass spectrometry [9-20]. However, no method of analysis for the quality control of CSBPT has been established.

In this study, a simultaneous quantitative analysis method was developed using 18 selected marker analytes for the quality evaluation of CSBPT based on HPLC separation combined with photodiode array detection (PDA). The selected components were prim$O$-glucosylcimifugin (3) and 5-O-methylvisammioside (9) from Saposhnikoviae Radix, oxypeucedanin hydrate (13) from Angelicae Dahuricae, arctigenin (11) and arctiin (16) from Forsythiae Fructus, baicalin (12), wogonoside (14), and baicalein (15) from Scutellariae Radix, ferulic acid (7) from Cnidii Rhizoma, pulegone (18) from Schizomepetae Spica, geniposide (1) from Gardeniae Fructus, coptisine chloride (2) and berberine chloride (4) from Coptidis Rhizoma, narirutin (8) and hesperidin (10) from Aurantii Fructus Immaturus, and liquiritin apioside (5), liquiritin (6), and glycyrrhizin (17) from Glycyrrhizae Radix et Rhizoma.

\section{Materials and Methods}

\subsection{Plant Materials}

Twelve crude plant materials, shown in Table S1, were purchased from a pharmaceutical manufacturer of herbal medicine (Kwangmyungdang Medicinal Herbs (KMH); Ulsan, Korea) in November 2017. The origins of these plant materials were confirmed by Dr. Seung-Yeol Oh, president of KMH, according to the guideline "The Dispensatory on the Visual and Organoleptic Examination of Herbal Medicine" [21]. Voucher specimens (2018KE69-1 to 2018KE63-12) of the crude plant material were deposited at the Herbal Medicine Research Division, Korea Institute of Oriental Medicine (KIOM, Daejeon, Korea).

\subsection{Chemicals and Reagents}

The standards used in this study were supplied from manufacturers specializing in standard compounds: compounds 1 (CAS No. 24512-62-7, $\geq 98.0 \%$ ), 2 (CAS No. 6020-1804, $\geq 98.0 \%$ ), 6 (CAS No. 551-15-5, 99.6\%), 7 (CAS No. 1135-24-6, 98.0\%), 12 (CAS No. 21967-419, 98.0\%), and 17 (CAS No. 1405-86-3, 99.4\%) from Fujifilm Wako Pure Chemical Co., Ltd. (Osaka, Japan); compound 3 (CAS No. 80681-45-4, 99.4\%) from ChemNorm Biotech Co., Ltd. (Wuhan, China); compounds 4 (CAS No. 633-65-8, 98.0\%) and 15 (CAS No. 491-67-8, 98.0\%) from KGaA (Darmstadt, Germany); compounds 5 (CAS No. 74639-14-8, $\geq 98.0 \%$ ), 11 (CAS No. 7770-78-7, 99.4\%), and 16 (CAS No. 20362-31-6, 98.1\%) from Shanghai Sunny Biotech Co., Ltd. (Shanghai, China); compounds 8 (CAS No. 14259-46-2, 99.5\%), 9 (CAS No. 84272-85-5, 99.9\%), 10 (CAS No. 520-26-3, 98.6\%), and 18 (CAS No. 89-82-7, 99.3\%) from 
Biopurify Phytochemicals (Chengdu, China); compound 13 (CAS No. 2643-85-8, 98.0\%) from ChemFaces Biochemical Co., Ltd. (Wuhan, China); and compound 14 (CAS No. 51059-44-0, 98.2\%) from Tauto Biotech (Shanghai, China). HPLC-grade acetonitrile, water, and trifluoroacetic acid $(\geq 99.0 \%)$, and ACS regent-grade formic acid $(\geq 98.0 \%)$, glacial acetic acid $(\geq 100.0 \%)$, and phosphoric acid $(85.0 \%)$ were used in the mobile phase, and they were purchased from JT Baker (Phillipsburg, NJ, USA), Merck KGaA (Darmstadt, Germany), and Fujifilm Wako Pure Chemical Co., Ltd. (Osaka, Japan), respectively. Methanol and dimethyl sulfoxide $(99.7 \%)$ used to prepare the test and standard solutions were purchased from JT Baker (Phillipsburg, NJ, USA) and Merck KGaA (Darmstadt, Germany), respectively.

\subsection{Preparation of CSBPT Water Extract}

CSBPT water extract was obtained from a formulation with the following weight ratio (total 5000 g): Saposhnikoviae Radix (500.7 g), Angelicae Dahuricae Radix (546.2 g), Forsythiae Fructus (546.2 g), Platycodonis Radix (546.2 g), Scutellariae Radix (477.1 g), Cnidii Rhizoma (477.1 g), Schizonepetae Spica (340.5 g), Gardeniae Fructus (340.5 g), Coptidis Rhizoma (340.5 g), Aurantii Fructus Immaturus (340.5 g), Menthae Herba (340.5 g), and Glycyrrhizae Radix et Rhizoma (204.0 g), and it was prepared in KIOM according to the extraction method detailed in the previous study using an electric extractor, COSMOS-660 (Kyungseo E \& P, Incheon, Korea) [22,23]. The prepared aqueous extract was lyophilized to obtain $1410.0 \mathrm{~g}$ of powder.

\subsection{Preparation of Test Solution and Standard Solution for HPLC-PDA}

To perform quantitative analysis on the 18 marker components of CSBPT using HPLCPDA, a test solution was prepared at a concentration of $10 \mathrm{mg} / \mathrm{mL}$ using $70 \%$ methanol. The prepared solution was extracted using an ultra-sonicator at room temperature for $60 \mathrm{~min}$ and then filtered with a $0.2 \mu \mathrm{m}$ polypropylene (GHP) membrane (Pall Life Sciences, Ann Arbor, MI, USA).

Each of the standard solution for compounds 1-18 was prepared at a concentration of $1000 \mu \mathrm{g} / \mathrm{mL}$ using either methanol or DMSO-methanol solution (1:1), and then stored at approximately $4{ }^{\circ} \mathrm{C}$ until use.

\subsection{HPLC Equipment and Chromatographic Separation Conditions}

The simultaneous analysis of the 18 marker compounds for quality evaluation of CSBPT was performed by using a modification of the previous methods [24,25]. A Shimadzu Prominence LC-20A series (Kyoto, Japan) HPLC system was used that combines a PDA for detection of the analyte and LabSolution software (Version 5.53, SP3, Kyoto, Japan) for data acquisition and processing. Other detailed analysis data are presented in Table S2.

\subsection{Validation of the Analytical Method}

The established HPLC assay was verified with respect to linearity, range, limit of detection (LOD), limit of quantification (LOQ), accuracy (recovery), and precision tests according to the International Conference on Harmonisation guidance [26]. Linearity of the response for the analysis of compounds 1-18 was evaluated based on the coefficient of determination $\left(r^{2}\right)$ of the calibration curve prepared at different concentrations $(0.31-20 \mu \mathrm{g} / \mathrm{mL}$ for compounds $7, \mathbf{8}, \mathbf{1 3}, \mathbf{1 4}$, and 18; $0.78-50 \mu \mathrm{g} / \mathrm{mL}$ for compounds $\mathbf{1}-\mathbf{3}, \mathbf{5}, \mathbf{6}, \mathbf{9}, \mathbf{1 1}, \mathbf{1 2}$, and $15-17 ; 1.25-80 \mu \mathrm{g} / \mathrm{mL}$ for compound 4 ; and $1.56-100 \mu \mathrm{g} / \mathrm{mL}$ for compound 10$)$.

Accuracy was assessed by conducting extraction recovery tests using the standard addition method.

The precision of the assay was evaluated by plotting the relative standard deviation (RSD) of each parameter, such as repeatability and intra- and inter-day precisions. In other words, the repeatability was evaluated by the RSD value of the retention time and peak area of each marker component after six repeated measurements of a standard solution. 
Intra- and inter-day precisions were assessed by calculating the RSD value based on the data measured on one day and on three consecutive days, respectively.

System suitability in the established assay was verified by confirming parameters such as the capacity factor $\left(k^{\prime}\right)$, selectivity factor $(\alpha)$, resolution $(R s)$, number of theoretical plates $(N)$, and tailing factor $(T f)$ values [27].

\section{Results and Discussion}

\subsection{Confirmation of the Main Component in Each Raw Herbal Medicine}

For the selection of marker components for the quality evaluation of CSBPT, we analyzed and confirmed the main components of 12 raw herbs as follows: prim-Oglucosylcimifugin and 5-O-methylvisammioside from Saposhnikoviae Radix; imperatorin, isoimperatorin, oxypeucedanin, and oxypeucedanin hydrate from Angelicae Dahuricae; arctigenin and arctiin from Forsythiae Fructus; platycodin D and platycodin D2 from Platycodonis Radix; baicalin, wogonoside, baicalein, and wogonin from Scutellariae Radix; ferulic acid from Cnidii Rhizoma; pulegone from Schizonepetae Spica; gardenoside and geniposide from Gardeniae Fructus; berberine chloride, coptisine chloride, and palmatine chloride from Coptidis Rhizoma; narirutin, naringin, hesperidin, and neohesperidin from Aurantii Fructus Immaturus; menthol and menthone from Menthae Herba; and glycyrrhizin, liquiritigenin, liquiritin apioside, and liquiritin from Glycyrrhizae Radix et Rhizoma. The presence of these herbal medicines and constituents was confirmed by HPLC using a distilled water-acetonitrile mobile phase system containing formic acid (Supplementary Materials Figure S1).

\subsection{Selection of Marker Components for Quality Assessment of CSBPT}

The major components that were identified in each herbal medicine are described in Section 3.1 and were also identified in the CSBPT sample. Thus, the 18 compounds were detected in the CSBPT sample among a total 31 components, as shown in Figure S2. These 18 compounds were selected as suitable marker components for the quality control of CSBPT; the chemical structures of the components are shown in Figure S3.

\subsection{Optimization of HPLC Chromatographic Separation Conditions}

For efficient chromatographic separation of compounds 1-18, various parameters, such as make and type of column [SunFire ${ }^{\mathrm{TM}}$ (Waters, Milford, MA, USA), Gemini (Phenomenex, Torrance, CA, USA), Capcellpak UG120 (Shiseido, Tokyo, Japan), Quasar ${ }^{\mathrm{TM}}$ SPP (PerkinElmer, Shelton, CT, USA), and INNO (YoungJin Biochrom Co., Seongnam Korea)], acids (formic acid, trifluoroacetic acid, acetic acid, and phosphoric acid), and column temperatures $\left(30,35,40\right.$, and $\left.45^{\circ} \mathrm{C}\right)$, were compared. Optimal analysis was achieved with a SunFire $\mathrm{C}_{18}$ column $(4.6 \times 250 \mathrm{~mm}, 5 \mu \mathrm{m})$, a column temperature of $40^{\circ} \mathrm{C}$, and two-component mobile phase system of distilled water-acetonitrile, both containing $0.1 \%$ $(v / v)$ formic acid. Under the optimized HPLC conditions, compounds 1-18 were baseline separated with resolution values $\geq 3.85$, with HPLC run-times within $45 \mathrm{~min}$ (Figure 1 and Table S3). 

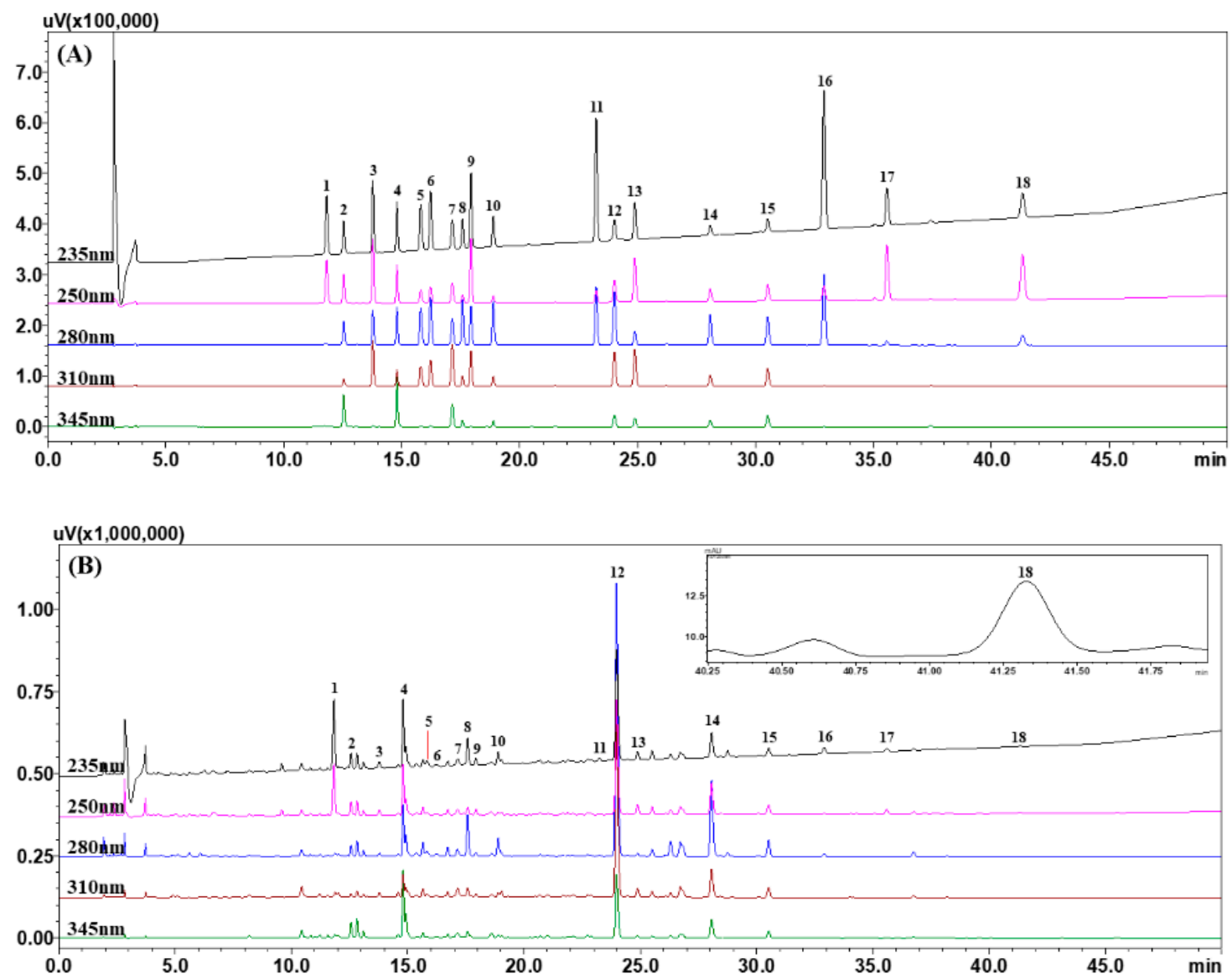

Figure 1. HPLC chromatograms of the standard solution (A) and test solution for quantification of $70 \%$ methanol extract of Cheongsangbangpung-tang (CSBPT) sample (B). Geniposide (1), coptisine $\mathrm{Cl}$ (2), prim-O-glucosylcimifugin (3), berberine $\mathrm{Cl}$ (4), liquiritin apioside (5), liquiritin (6), ferulic acid (7), narirutin (8), 5-O-methylvisammioside (9), hesperidin (10), arctigenin (11), baicalin (12), oxypeucedanin hydrate (13), wogonoside (14), baicalein (15), arctiin (16), glycyrrhizin (17), and pulegone (18).

\subsection{Method Validation of the Developed HPLC Analytical Method}

For quality control of CSBPT based on compounds 1-18, the optimized analysis method was verified by evaluating factors such as system suitability, linearity, LOD, LOQ, and precision (repeatability, and intra- and inter-day precisions). The values of the system suitability factors $k^{\prime}, \alpha, N, R s$, and $T f$ were calculated to be 3.23-13.80, 1.02-1.18, 247,354.40$1,161,300.13,3.85-36.85$, and 1.01-1.22, respectively (Table S3). The $r^{2}$ values of compounds 1-18 calculated from the corresponding calibration curves were $\geq 0.9996$, which confirmed the excellent linearity of the analysis assay. The LOD and LOQ values of compounds 1-18 were $0.07-0.36 \mu \mathrm{g} / \mathrm{mL}$ and $0.21-1.10 \mu \mathrm{g} / \mathrm{mL}$, respectively. Regression equation, $r^{2}$, LOD, and LOQ data are presented in Table 1 . The extraction recovery of compounds 1-18 for evaluation of the accuracy of the developed assay was $97.03-104.04 \%$, and the RSD $(\%)$ value was $<3.00$ (Table 2). The RSD values of repeatability, and intra- and inter-day precisions of compounds 1-18 were $0.01-3.09 \%$, showing good precision results (Table 3 ). These data confirmed that the developed HPLC assay was suitable for the quality control of CSBPT. 
Table 1. Linear range, regression equation, $r^{2}$, limit of detection (LOD), and limit of quantification (LOQ) values for HPLC analysis of compounds 1-18 $(n=3)$.

\begin{tabular}{|c|c|c|c|c|c|c|}
\hline Analyte & $\begin{array}{l}\text { Detection } \\
(\mathrm{nm})\end{array}$ & $\begin{array}{c}\text { Linear Range } \\
(\mu \mathrm{g} / \mathrm{mL})\end{array}$ & $\begin{array}{c}\text { Regression Equation }^{a} \\
y=a x+b\end{array}$ & $r^{2}$ & LOD $(\mu \mathrm{g} / \mathrm{mL})$ & LOQ $(\mu \mathrm{g} / \mathrm{mL})$ \\
\hline 1 & 235 & $0.78-50$ & $y=22,979.62 x+2649.51$ & 1.0000 & 0.09 & 0.28 \\
\hline 2 & 355 & $0.78-50$ & $y=39,831.26 x-5918.67$ & 0.9996 & 0.20 & 0.60 \\
\hline 3 & 300 & $0.78-50$ & $y=19,703.59 x+3163.52$ & 1.0000 & 0.16 & 0.48 \\
\hline 4 & 345 & $1.25-80$ & $y=47,572.65 x+12858.14$ & 1.0000 & 0.31 & 0.95 \\
\hline 5 & 275 & $0.78-50$ & $y=17,486.99 x+3289.39$ & 1.0000 & 0.20 & 0.61 \\
\hline 6 & 275 & $0.78-50$ & $y=21,989.62 x+4049.96$ & 1.0000 & 0.20 & 0.60 \\
\hline 7 & 320 & $0.31-20$ & $y=65,199.18 x+4792.95$ & 1.0000 & 0.08 & 0.25 \\
\hline 8 & 280 & $0.31-20$ & $y=17,012.50 x+1238.65$ & 1.0000 & 0.07 & 0.21 \\
\hline 9 & 290 & $0.78-50$ & $y=17,739.58 x+3428.71$ & 1.0000 & 0.20 & 0.60 \\
\hline 10 & 280 & $1.56-100$ & $y=16,112.67 x+6984.59$ & 1.0000 & 0.36 & 1.10 \\
\hline 11 & 280 & $0.78-50$ & $y=7271.72 x+1344.77$ & 1.0000 & 0.20 & 0.60 \\
\hline 12 & 275 & $0.78-50$ & $y=38,381.96 x+6880.25$ & 1.0000 & 0.20 & 0.61 \\
\hline 13 & 310 & $0.31-20$ & $y=53,174.23 x+3697.05$ & 1.0000 & 0.09 & 0.28 \\
\hline 14 & 275 & $0.31-20$ & $y=48,111.17 x+3026.11$ & 1.0000 & 0.09 & 0.28 \\
\hline 15 & 275 & $0.78-50$ & $y=57,618.21 x+3704.23$ & 1.0000 & 0.15 & 0.45 \\
\hline 16 & 280 & $0.78-50$ & $y=10,789.77 x+2166.35$ & 1.0000 & 0.20 & 0.61 \\
\hline 17 & 250 & $0.78-50$ & $y=8540.20 x+1806.55$ & 1.0000 & 0.21 & 0.64 \\
\hline 18 & 250 & $0.31-20$ & $y=18,446.27 x+1845.86$ & 1.0000 & 0.07 & 0.22 \\
\hline
\end{tabular}

a $y$ : peak area of compounds; $x$ : concentration $(\mu \mathrm{g} / \mathrm{mL})$ of compounds.

Table 2. Extract recovery test of compounds 1-18 in the developed analytical assay.

\begin{tabular}{|c|c|c|c|c|c|}
\hline Analyte & $\begin{array}{c}\text { Measured } \\
\text { Amount }(\mu \mathrm{g} / \mathrm{mL})\end{array}$ & $\begin{array}{l}\text { Found Amount } \\
\qquad(\mu \mathrm{g} / \mathrm{mL})\end{array}$ & Recovery (\%) & SD & RSD (\%) \\
\hline \multirow{4}{*}{1} & 1.00 & 1.01 & 100.81 & 1.73 & 1.72 \\
\hline & 2.00 & 1.99 & 99.41 & 0.94 & 0.95 \\
\hline & 4.00 & 3.93 & 98.23 & 1.17 & 1.19 \\
\hline & 2.00 & 2.07 & 103.52 & 0.98 & 0.94 \\
\hline \multirow[t]{3}{*}{2} & 4.00 & 3.99 & 99.70 & 1.00 & 1.00 \\
\hline & 8.00 & 8.27 & 103.36 & 0.59 & 0.57 \\
\hline & 1.00 & 1.02 & 101.52 & 1.50 & 1.48 \\
\hline \multirow[t]{3}{*}{3} & 2.00 & 1.99 & 99.58 & 0.36 & 0.36 \\
\hline & 4.00 & 3.96 & 99.07 & 0.82 & 0.83 \\
\hline & 4.00 & 4.04 & 100.90 & 2.80 & 2.77 \\
\hline \multirow[t]{3}{*}{4} & 10.00 & 9.92 & 99.24 & 2.97 & 2.99 \\
\hline & 20.00 & 19.41 & 97.03 & 1.13 & 1.17 \\
\hline & 2.00 & 1.98 & 99.19 & 1.86 & 1.88 \\
\hline \multirow[t]{3}{*}{5} & 5.00 & 4.97 & 99.45 & 1.11 & 1.12 \\
\hline & 10.00 & 9.91 & 99.13 & 1.40 & 1.41 \\
\hline & 1.00 & 1.00 & 100.31 & 1.24 & 1.24 \\
\hline \multirow[t]{3}{*}{6} & 2.00 & 2.01 & 100.43 & 1.48 & 1.47 \\
\hline & 4.00 & 4.04 & 101.04 & 0.85 & 0.84 \\
\hline & 1.00 & 1.01 & 100.56 & 1.79 & 1.78 \\
\hline \multirow[t]{3}{*}{7} & 2.00 & 1.99 & 99.73 & 0.67 & 0.67 \\
\hline & 4.00 & 3.91 & 97.79 & 0.44 & 0.45 \\
\hline & 1.00 & 1.04 & 104.04 & 2.36 & 2.27 \\
\hline \multirow[t]{2}{*}{8} & 2.00 & 1.96 & 98.25 & 2.56 & 2.60 \\
\hline & 4.00 & 3.97 & 99.32 & 1.50 & 1.51 \\
\hline
\end{tabular}


Table 2. Cont.

\begin{tabular}{|c|c|c|c|c|c|}
\hline Analyte & $\begin{array}{c}\text { Measured } \\
\text { Amount }(\mu \mathrm{g} / \mathrm{mL})\end{array}$ & $\begin{array}{l}\text { Found Amount } \\
(\mu \mathrm{g} / \mathrm{mL})\end{array}$ & Recovery (\%) & SD & RSD (\%) \\
\hline \multirow{4}{*}{9} & 1.00 & 1.00 & 99.65 & 1.40 & 1.41 \\
\hline & 2.00 & 2.02 & 100.84 & 1.34 & 1.33 \\
\hline & 4.00 & 4.06 & 101.62 & 0.84 & 0.83 \\
\hline & 5.00 & 5.13 & 102.63 & 2.36 & 2.30 \\
\hline \multirow[t]{3}{*}{10} & 12.50 & 12.79 & 102.29 & 1.23 & 1.20 \\
\hline & 25.00 & 25.09 & 100.36 & 1.36 & 1.36 \\
\hline & 2.00 & 1.97 & 98.37 & 2.86 & 2.90 \\
\hline \multirow{3}{*}{11} & 5.00 & 4.96 & 99.16 & 1.60 & 1.61 \\
\hline & 10.00 & 9.86 & 98.57 & 1.01 & 1.03 \\
\hline & 3.00 & 2.96 & 98.74 & 0.97 & 0.98 \\
\hline \multirow[t]{3}{*}{12} & 7.50 & 7.42 & 98.89 & 0.82 & 0.83 \\
\hline & 15.00 & 15.44 & 102.95 & 0.72 & 0.70 \\
\hline & 1.00 & 1.01 & 100.80 & 1.49 & 1.47 \\
\hline \multirow[t]{3}{*}{13} & 2.00 & 2.03 & 101.35 & 0.54 & 0.53 \\
\hline & 4.00 & 4.05 & 101.20 & 0.67 & 0.66 \\
\hline & 1.00 & 1.01 & 100.61 & 1.83 & 1.82 \\
\hline \multirow[t]{3}{*}{14} & 2.00 & 2.03 & 101.74 & 0.90 & 0.89 \\
\hline & 4.00 & 4.14 & 103.59 & 0.88 & 0.85 \\
\hline & 2.00 & 1.99 & 99.45 & 1.20 & 1.21 \\
\hline \multirow[t]{3}{*}{15} & 5.00 & 4.96 & 99.14 & 1.61 & 1.62 \\
\hline & 10.00 & 9.82 & 98.25 & 1.79 & 1.82 \\
\hline & 2.00 & 2.04 & 101.96 & 0.89 & 0.87 \\
\hline \multirow[t]{3}{*}{16} & 4.00 & 4.06 & 101.47 & 1.44 & 1.42 \\
\hline & 8.00 & 8.04 & 100.53 & 1.64 & 1.63 \\
\hline & 3.00 & 3.10 & 103.47 & 2.29 & 2.21 \\
\hline \multirow[t]{3}{*}{17} & 7.50 & 7.53 & 100.37 & 1.18 & 1.18 \\
\hline & 15.00 & 14.99 & 99.90 & 0.34 & 0.34 \\
\hline & 1.00 & 0.99 & 98.85 & 1.91 & 1.93 \\
\hline \multirow[t]{2}{*}{18} & 2.00 & 2.02 & 101.14 & 1.02 & 1.01 \\
\hline & 4.00 & 4.10 & 102.45 & 0.57 & 0.56 \\
\hline
\end{tabular}

Table 3. Precision validation of compounds 1-18 in the developed analytical assay.

\begin{tabular}{|c|c|c|c|c|c|c|c|c|c|}
\hline \multirow[b]{2}{*}{ Analyte } & \multirow[b]{2}{*}{$\begin{array}{l}\text { Conc. } \\
(\mu \mathrm{g} / \mathrm{mL})\end{array}$} & \multicolumn{3}{|c|}{ Intra-Day $(n=5)$} & \multicolumn{3}{|c|}{ Inter-Day $(n=5)$} & \multicolumn{2}{|c|}{ Repeatability $(n=6)$} \\
\hline & & $\begin{array}{c}\text { Observed } \\
\text { Conc. } \\
(\mu \mathrm{g} / \mathrm{mL})\end{array}$ & $\begin{array}{c}\text { Precision } \\
\quad(\%)^{a}\end{array}$ & $\begin{array}{c}\text { Accuracy } \\
(\%)\end{array}$ & $\begin{array}{c}\text { Observed } \\
\text { Conc. } \\
(\mu \mathrm{g} / \mathrm{mL})\end{array}$ & $\begin{array}{l}\text { Precision } \\
\text { (\%) }\end{array}$ & $\begin{array}{c}\text { Accuracy } \\
(\%)\end{array}$ & $\begin{array}{l}\text { RSD (\%) of } \\
\text { Retention } \\
\text { Time }\end{array}$ & $\begin{array}{c}\text { RSD }(\%) \\
\text { of Peak } \\
\text { Area }\end{array}$ \\
\hline \multirow{4}{*}{1} & 12.50 & 12.73 & 0.91 & 101.84 & 12.80 & 0.88 & 102.39 & \multirow{3}{*}{0.05} & \multirow{4}{*}{0.52} \\
\hline & 25.00 & 25.91 & 1.40 & 103.64 & 25.99 & 1.24 & 103.96 & & \\
\hline & 50.00 & 51.13 & 0.36 & 102.25 & 51.25 & 0.68 & 102.51 & & \\
\hline & 12.50 & 12.56 & 0.75 & 100.44 & 12.95 & 2.67 & 103.58 & \multirow{3}{*}{0.05} & \\
\hline \multirow[t]{3}{*}{2} & 25.00 & 24.99 & 1.23 & 99.98 & 25.41 & 2.21 & 101.64 & & \multirow[t]{2}{*}{0.16} \\
\hline & 50.00 & 51.03 & 2.81 & 102.05 & 50.98 & 2.69 & 101.97 & & \\
\hline & 12.50 & 12.97 & 1.49 & 103.79 & 13.33 & 2.54 & 106.62 & \multirow{3}{*}{0.04} & \multirow{4}{*}{0.52} \\
\hline \multirow[t]{3}{*}{3} & 25.00 & 26.05 & 1.65 & 104.18 & 26.57 & 2.56 & 106.28 & & \\
\hline & 50.00 & 51.50 & 1.51 & 103.00 & 51.72 & 1.79 & 103.44 & & \\
\hline & 20.00 & 20.72 & 1.28 & 103.62 & 21.30 & 2.65 & 106.52 & \multirow{4}{*}{0.03} & \\
\hline \multirow[t]{3}{*}{4} & 40.00 & 41.64 & 1.67 & 104.11 & 42.25 & 2.10 & 105.63 & & \multirow[t]{3}{*}{0.35} \\
\hline & 80.00 & 82.13 & 1.11 & 102.66 & 82.43 & 1.40 & 103.03 & & \\
\hline & 12.50 & 13.09 & 2.00 & 104.74 & 13.41 & 2.70 & 107.26 & & \\
\hline \multirow[t]{3}{*}{5} & 25.00 & 26.27 & 2.10 & 105.09 & 26.63 & 2.27 & 106.53 & \multirow[t]{2}{*}{0.03} & \multirow[t]{3}{*}{0.46} \\
\hline & 50.00 & 51.73 & 1.50 & 103.47 & 51.85 & 1.46 & 103.70 & & \\
\hline & 12.50 & 13.06 & 1.82 & 104.48 & 13.34 & 2.52 & 106.69 & \multirow{3}{*}{0.03} & \\
\hline \multirow[t]{2}{*}{6} & 25.00 & 26.27 & 1.96 & 105.06 & 26.59 & 2.01 & 106.34 & & \multirow[t]{2}{*}{0.41} \\
\hline & 50.00 & 51.67 & 1.31 & 103.34 & 51.73 & 1.20 & 103.46 & & \\
\hline
\end{tabular}


Table 3. Cont.

\begin{tabular}{|c|c|c|c|c|c|c|c|c|c|}
\hline \multirow[b]{2}{*}{ Analyte } & \multirow[b]{2}{*}{$\begin{array}{c}\text { Conc. } \\
(\mu \mathrm{g} / \mathrm{mL})\end{array}$} & \multicolumn{3}{|c|}{ Intra-Day $(n=5)$} & \multicolumn{3}{|c|}{ Inter-Day $(n=5)$} & \multicolumn{2}{|c|}{ Repeatability $(n=6)$} \\
\hline & & $\begin{array}{c}\text { Observed } \\
\text { Conc. } \\
(\mu \mathrm{g} / \mathrm{mL})\end{array}$ & $\begin{array}{l}\text { Precision } \\
\quad(\%)^{a}\end{array}$ & $\begin{array}{c}\text { Accuracy } \\
(\%)\end{array}$ & $\begin{array}{l}\text { Observed } \\
\text { Conc. } \\
(\mu \mathrm{g} / \mathrm{mL})\end{array}$ & $\begin{array}{l}\text { Precision } \\
\quad(\%)\end{array}$ & $\begin{array}{c}\text { Accuracy } \\
(\%)\end{array}$ & $\begin{array}{l}\text { RSD (\%) of } \\
\text { Retention } \\
\text { Time }\end{array}$ & $\begin{array}{c}\text { RSD (\%) } \\
\text { of Peak } \\
\text { Area }\end{array}$ \\
\hline \multirow{4}{*}{7} & 5.00 & 5.20 & 1.44 & 104.07 & 5.34 & 2.33 & 106.90 & \multirow{3}{*}{0.03} & \multirow{4}{*}{0.37} \\
\hline & 10.00 & 10.49 & 1.53 & 104.86 & 10.66 & 2.34 & 106.62 & & \\
\hline & 20.00 & 20.65 & 1.39 & 103.24 & 20.74 & 1.54 & 103.69 & & \\
\hline & 5.00 & 5.21 & 1.87 & 104.22 & 5.37 & 3.04 & 107.48 & \multirow{3}{*}{0.03} & \\
\hline \multirow[t]{3}{*}{8} & 10.00 & 10.50 & 1.69 & 104.97 & 10.68 & 2.43 & 106.78 & & \multirow[t]{3}{*}{0.40} \\
\hline & 20.00 & 20.68 & 1.36 & 103.41 & 20.79 & 1.65 & 103.97 & & \\
\hline & 12.50 & 12.89 & 1.73 & 103.12 & 13.20 & 2.33 & 105.63 & \multirow{3}{*}{0.03} & \\
\hline \multirow[t]{3}{*}{9} & 25.00 & 26.02 & 2.05 & 104.09 & 26.41 & 2.37 & 105.62 & & \multirow{3}{*}{0.25} \\
\hline & 50.00 & 51.04 & 1.42 & 102.08 & 51.29 & 1.60 & 102.58 & & \\
\hline & 25.00 & 26.14 & 1.83 & 104.56 & 26.96 & 3.05 & 107.85 & \multirow{3}{*}{0.03} & \\
\hline \multirow[t]{3}{*}{10} & 50.00 & 52.56 & 1.77 & 105.12 & 53.45 & 2.41 & 106.90 & & \multirow[t]{3}{*}{0.35} \\
\hline & 100.00 & 103.20 & 1.43 & 103.20 & 103.79 & 1.67 & 103.79 & & \\
\hline & 12.50 & 13.09 & 1.79 & 104.73 & 13.52 & 3.09 & 108.17 & \multirow{4}{*}{0.02} & \\
\hline \multirow[t]{3}{*}{11} & 25.00 & 26.44 & 1.98 & 105.75 & 26.86 & 2.38 & 107.44 & & \multirow[t]{3}{*}{0.39} \\
\hline & 50.00 & 52.21 & 1.52 & 104.43 & 52.41 & 1.69 & 104.82 & & \\
\hline & 12.50 & 12.85 & 1.67 & 102.80 & 13.29 & 3.07 & 106.32 & & \\
\hline \multirow[t]{3}{*}{12} & 25.00 & 26.10 & 1.32 & 104.42 & 26.39 & 1.69 & 105.55 & \multirow{3}{*}{0.02} & \multirow[t]{3}{*}{0.43} \\
\hline & 50.00 & 51.32 & 1.39 & 102.63 & 51.66 & 1.60 & 103.31 & & \\
\hline & 5.00 & 5.22 & 1.74 & 104.38 & 5.35 & 2.53 & 107.03 & & \\
\hline \multirow[t]{3}{*}{13} & 10.00 & 10.52 & 1.94 & 105.17 & 10.63 & 2.02 & 106.32 & \multirow[t]{3}{*}{0.02} & 0.33 \\
\hline & 20.00 & 20.67 & 1.39 & 103.34 & 20.69 & 1.23 & 103.46 & & \\
\hline & 5.00 & 5.19 & 1.73 & 103.87 & 5.37 & 3.08 & 107.31 & & \\
\hline 14 & 10.00 & 10.49 & 1.82 & 104.91 & 10.66 & 2.37 & 106.58 & 0.02 & 0.35 \\
\hline & 20.00 & 20.62 & 1.30 & 103.09 & 20.74 & 1.54 & 103.68 & & \\
\hline & 12.50 & 12.87 & 1.50 & 102.94 & 13.13 & 2.83 & 105.03 & & \\
\hline 15 & 25.00 & 26.04 & 0.99 & 104.17 & 26.18 & 1.22 & 104.71 & 0.02 & 0.39 \\
\hline & 50.00 & 51.45 & 1.12 & 102.90 & 51.19 & 0.93 & 102.38 & & \\
\hline & 12.50 & 13.07 & 1.81 & 104.60 & 13.47 & 2.95 & 107.79 & & \\
\hline 16 & 25.00 & 26.28 & 1.92 & 105.13 & 26.71 & 2.43 & 106.83 & 0.02 & 0.38 \\
\hline & 50.00 & 51.65 & 1.40 & 103.31 & 51.92 & 1.65 & 103.84 & & \\
\hline & 12.50 & 13.07 & 1.83 & 104.55 & 13.36 & 2.06 & 106.85 & & \\
\hline 17 & 25.00 & 26.20 & 1.74 & 104.82 & 26.50 & 1.79 & 106.02 & 0.01 & 0.32 \\
\hline & 50.00 & 51.54 & 1.32 & 103.08 & 51.83 & 1.59 & 103.67 & & \\
\hline & 5.00 & 5.18 & 1.18 & 103.58 & 5.31 & 2.22 & 106.24 & & \\
\hline 18 & 10.00 & 10.41 & 1.33 & 104.06 & 10.55 & 1.83 & 105.53 & 0.01 & 0.27 \\
\hline & 20.00 & 20.52 & 1.06 & 102.60 & 20.57 & 1.02 & 102.87 & & \\
\hline
\end{tabular}

\subsection{Quantification of Compounds $\mathbf{1}-\mathbf{1 8}$ in CSBPT Samples}

By using the developed and verified HPLC analysis method, quantification of the selected 18 marker components was performed for quality assessment of CSBPT samples. Quantification of compounds 1-18 was achieved by detection at the maximum absorption wavelength in the UV spectrum of each component. The amount of compounds 1-18 in the freeze-dried CSBPT sample was found to be $0.27-18.31 \mathrm{mg} / \mathrm{g}$; among these analytes, compound 12, the major constituent of Scutellariae Radix, was identified as the most abundant (17.82-18.31 mg/g; Table 4). 
Table 4. Concentration of compounds 1-18 in CSBPT according to sample batches $(n=3)$.

\begin{tabular}{|c|c|c|c|c|c|c|}
\hline \multirow{3}{*}{ Analyte } & \multicolumn{6}{|c|}{ Batch No. } \\
\hline & \multicolumn{2}{|c|}{ I } & \multicolumn{2}{|c|}{ II } & \multicolumn{2}{|c|}{ III } \\
\hline & $\begin{array}{c}\text { Mean }(\mathrm{mg} / \mathrm{g}) \pm \\
\mathrm{SD}\left(\times 10^{-2}\right)\end{array}$ & RSD (\%) & $\begin{array}{c}\text { Mean }(\mathrm{mg} / \mathrm{g}) \pm \\
\mathrm{SD}\left(\times 10^{-2}\right)\end{array}$ & RSD (\%) & $\begin{array}{l}\text { Mean }(\mathrm{mg} / \mathrm{g}) \pm \\
\mathrm{SD}\left(\times 10^{-2}\right)\end{array}$ & RSD (\%) \\
\hline 1 & $5.33 \pm 1.02$ & 0.19 & $5.35 \pm 4.59$ & 0.86 & $5.41 \pm 3.41$ & 0.63 \\
\hline 2 & $0.70 \pm 0.45$ & 0.63 & $0.75 \pm 0.31$ & 0.41 & $0.71 \pm 0.19$ & 0.26 \\
\hline 3 & $0.60 \pm 0.87$ & 1.45 & $0.59 \pm 0.60$ & 1.02 & $0.59 \pm 0.18$ & 0.31 \\
\hline 4 & $2.30 \pm 3.72$ & 1.62 & $2.45 \pm 2.10$ & 0.86 & $2.30 \pm 1.70$ & 0.74 \\
\hline 5 & $1.16 \pm 1.41$ & 1.22 & $1.16 \pm 1.18$ & 1.01 & $1.14 \pm 0.97$ & 0.86 \\
\hline 6 & $0.54 \pm 0.45$ & 0.82 & $0.55 \pm 0.41$ & 0.75 & $0.54 \pm 0.25$ & 0.47 \\
\hline 7 & $0.50 \pm 0.20$ & 0.39 & $0.50 \pm 0.94$ & 1.87 & $0.50 \pm 0.29$ & 0.58 \\
\hline 8 & $4.55 \pm 0.42$ & 0.09 & $4.57 \pm 2.82$ & 0.62 & $4.56 \pm 1.21$ & 0.27 \\
\hline 9 & $0.60 \pm 0.32$ & 0.53 & $0.60 \pm 0.51$ & 0.84 & $0.60 \pm 0.24$ & 0.40 \\
\hline 10 & $2.51 \pm 1.45$ & 0.58 & $2.54 \pm 1.66$ & 0.65 & $2.51 \pm 1.54$ & 0.61 \\
\hline 11 & $1.11 \pm 1.04$ & 0.94 & $1.16 \pm 1.60$ & 1.38 & $1.15 \pm 2.10$ & 1.83 \\
\hline 12 & $17.82 \pm 7.34$ & 0.41 & $18.31 \pm 3.76$ & 0.21 & $18.19 \pm 9.31$ & 0.51 \\
\hline 13 & $0.46 \pm 0.03$ & 0.06 & $0.47 \pm 0.28$ & 0.60 & $0.47 \pm 0.23$ & 0.50 \\
\hline 14 & $4.40 \pm 1.65$ & 0.38 & $4.52 \pm 2.19$ & 0.48 & $4.48 \pm 1.05$ & 0.24 \\
\hline 15 & $1.02 \pm 2.23$ & 2.19 & $1.05 \pm 1.49$ & 1.42 & $0.99 \pm 2.01$ & 2.02 \\
\hline 16 & $0.82 \pm 0.20$ & 0.24 & $0.85 \pm 0.25$ & 0.30 & $0.82 \pm 0.72$ & 0.88 \\
\hline 17 & $1.51 \pm 0.82$ & 0.54 & $1.52 \pm 0.25$ & 0.16 & $1.48 \pm 0.29$ & 0.20 \\
\hline 18 & $0.27 \pm 0.08$ & 0.28 & $0.27 \pm 0.05$ & 0.17 & $0.27 \pm 0.07$ & 0.26 \\
\hline
\end{tabular}

\section{Conclusions}

We developed a simultaneous analysis method for selected compounds 1-18 that enables the quality assessment of CSBPT based on a common and widely-used HPLCPDA system. The developed assay was validated by evaluating linearity, LOD, LOQ, recovery, and precision, and it was successfully applied to the analysis of CSBPT samples. This HPLC-PDA analytical assay can be used as a framework to obtain basic data for quality assessment of CSBPT and other traditional Korean medicines in the future.

Supplementary Materials: The following are available online at https://www.mdpi.com/2076-3 417/11/1/14/s1, Figure S1: HPLC chromatogram of constituent herbal medicine and its major components. A: S. divaricate; B: A. dahurica; C: F. viridissima; D: P. grandiflorum; E: S. baicalensis; F: C. officinale; G: S. tenuifolia; H: G. jasminoides; I: C. japonica; J: C. aurantium; K: M. arvensis; and L: G. uralensis, Figure S2: HPLC chromatograms of the standard solution (A) and 70\% methanol extract of CSBPT sample (B). Gardenoside (1), geniposide (2), coptisine chloride (3), prim-O-glucosylcimifugin (4), berberine chloride (5), palmatine chloride (6), liquiritin apioside (7), liquiritin (8), ferulic acid (9), narirutin (10), 5-O-methylcisammisoide (11), naringin (12), hesperidin (13), neohesperidin (14), arctigenin (15), platycodin D2 (16), baicalin (17), platycodin D (18), liquiritigenin (19), oxypeucedanin hydrate (20), wogonoside (21), baicalein (22), menthole (23), arctiin (24), glycyrrhizin (25), wogonin (26), oxypeucedanin (27), pulegone (28), menthone (29), imperatoin (30), isoimperatorin (31), Figure S3: Chemical structures of compounds 1-18 in CSBPT, Table S1: Composition of CSBPT, Table S2: Chromatographic conditions for simultaneous quantification of compounds 1-18 in CSBPT, Table S3: System suitability of compounds 1-18.

Author Contributions: Conceptualization: C.-S.S. and H.-K.S.; performing experiments and analyzing data: C.-S.S.; writing —original draft preparation: C.-S.S.; funding acquisition: H.-K.S. All authors have read and agreed to the published version of the manuscript.

Funding: This research was supported by a grant from the Korea Institute of Oriental Medicine (No. KSN2013310).

Conflicts of Interest: The authors have declared no conflict of interest. 


\section{References}

1. Woo, C.Y.; Kim, D.C. Skin regeneration, anti-wrinkle, whitening and moisturizing effects of Cheongsangbangpung-tang aqueous extracts with cytotoxicity. J. Korean Obs. Gynecol. 2017, 30, 49-70.

2. Heo, J. Donguibogam; Namsandang: Seoul, Korea, 2007; p. 211.

3. Higaki, S.; Hasegawa, Y.; Morohashi, M.; Takayoshi, Y. The correlation of Kampo formulations and their ingredients on anti-bacterial activities against Propionibacterium acnes. J. Derm. 1995, 22, 4-9. [CrossRef] [PubMed]

4. Lee, E.S.; Seo, B.I. The antimicrobial activity of Cheongsangbangpungtang on Proteus sp. Korea J. Herbol. 2004, 19, 101-108.

5. Jo, H.; Oh, E.; Jee, S.; Seo, B. The antimicrobial activity of Cheongsangbangpungtang and its composition oriental medicines against Staphylococcus aureus. Korea J. Herbol. 2003, 18, 37-47.

6. Jin, K.S.; Lim, T.H.; Park, E.J.; Lee, H.J.; Han, K.H.; Na, W.K. Regulatory effect of atopic allergic reaction by Chungsangbangpoongtang. J. Korean Orient. Pediatr. 2004, 18, 61-76.

7. Saruwatari, J.; Takashima, A.; Yoshida, K.; Soraoka, H.; Ding, T.B.; Uchiyashiki, Y.; Tsuda, Y.; Imamura, M.; Oniki, K.; Miyata, K.; et al. Effects of Seijo-bofu-to, a traditional Japanese herbal medicine containing furanocoumarin derivatives, on the drug-metabolizing enzyme activities in healthy male volunteers. Basic Clin. Pharmacol. Toxicol. 2014, 115, 360-365. [CrossRef]

8. Yang, S.H.; Lin, Y.H.; Lin, J.R.; Chen, H.Y.; Hu, S.; Yang, Y.H.; Yang, Y.H.; Yang, Y.S.; Fang, Y.G. The efficacy and safety of a fixed combination of Chinese herbal medicine in chronic urticarial: A randomized, double-blind, placebo-controlled pilot study. Front. Pharmacol. 2018, 9, 1474. [CrossRef]

9. Kim, M.K.; Yang, D.H.; Jung, M.; Jung, E.H.; Eom, H.Y.; Suh, J.H.; Min, J.W.; Kim, U.; Min, H.; Kim, J.; et al. Simultaneous determination of chromones and coumarins in Radix Saposhnikoviae by high performance liquid chromatography with diode array and tandem mass detectors. J. Chromatogr. A 2011, 1218, 6319-6330. [CrossRef]

10. Li, B.; Zhang, X.; Wang, J.; Zhang, L.; Gao, B.; Shi, S.; Wang, X.; Li, J.; Tu, P. Simultaneous characterisation of fifty coumarins from the roots of Angelica dahurica by off-line tow-dimensional high-performance liquid chromatography coupled with electrospray ionisation tandem mass spectrometry. Phytochem. Anal. 2014, 25, 229-240. [CrossRef]

11. Won, T.H.; Liao, L.; Lee, S.H.; Son, J.K.; Shin, J. Comparative analyses of bioactive constituents from Forsythia suspensa and Forsythia viridissima by HPLC-DAD. Nat. Prod. Sci. 2011, 17, 328-336.

12. Kim, H.K.; Choi, J.S.; Yoo, D.S.; Choi, Y.H.; Yon, G.H.; Hong, K.S.; Lee, B.H.; Kim, H.J.; Kim, E.J.; Park, B.K.; et al. HPLC analysis of saponins in Platycodi Radix. Kor. J. Pharmacol. 2007, 38, 192-1296.

13. Tong, L.; Wan, M.; Zhang, L.; Zhu, Y.; Sun, H.; Bi, K. Simultaneous determination of baicalin, wogonoside, baicalein, wogonin, oroxylin A and chrysin of Radix Scutellariae extract in rat plasma by liquid chromatography tanderm mass spectrometry. J. Pharmacol. BioMed Anal. 2012, 70, 6-12. [CrossRef] [PubMed]

14. Baek, M.E.; Seong, G.U.; Lee, Y.J.; Won, J.H. Quantitative analysis for the quality evaluation of active ingredients in Cnidium Rhizome. Yakhak Hoeji 2016, 60, 227-234. [CrossRef]

15. Shan, M.Q.; Qian, Y.; Yu, S.; Guo, S.C.; Zhang, L.; Ding, A.W.; Wu, Q.N. Anti-inflammatory effect of volatile oil from Schizonepeta tenuifolia on carrageenin-induced pleurisy in rats and its application to study of appropriate harvesting time coupled with multi-attribute comprehensive index method. J. Ethnopharmacol. 2016, 194, 580-586. [CrossRef] [PubMed]

16. Lee, E.J.; Hong, J.K.; Whang, W.K. Simultaneous determination of bioactive marker compounds from Gardeniae Fructus by high performance liquid chromatography. Arch. Pharmacol. Res. 2014, 37, 992-1000. [CrossRef]

17. Lv, X.; Li, Y.; Tang, C.; Zhang, Y.; Zhang, J.; Fan, G. Integration of HPLC-based fingerprint and quantitative analyses for differentiation botanical species and geographical growing origins of Rhizoma Coptidis. Pharmacol. Biol. 2016, 54, 3264-3271. [CrossRef]

18. Wang, C.; Pan, Y.; Fan, G.; Chai, Y.; Wu, Y. Application of an efficient strategy based on MAE, HPLC-DAD-MS/MS and HSCCC for the rapid extraction, identification, separation and purification of flavonoids from Fructus Aurantii Immaturus. BioMed Chromatogr. 2010, 24, 235-244. [CrossRef]

19. Shin, K.E.; Park, H.K. Changes of essential oils from Mentha piperita L. influenced by various cultivation conditions and harvesting time. Korea J. Food Sci. Technol. 1994, 26, 512-519.

20. Zhou, S.; Cao, J.; Qiu, F.; Kong, W.; Yang, S.; Yang, M. Simultaneous determination of five bioactive components in Radix Glycyrrhizae by pressurized liquid extraction combined with UPLC-PDA and UPLC/ESI-QTOF-MS confirmation. Phytochem. Anal. 2013, 24, 527-533. [CrossRef]

21. Lee, K.H. The Dispensatory on the Visual and Organoleptic Examination of Herbal Medicine; National Institute of Food and Drug Safety Evaluation: Seoul, Korea, 2013; pp. 24-728.

22. Seo, C.S.; Yoo, S.R.; Jeong, S.J.; Ha, H. Quantification of the constituents of the traditional Korea medicine, Samryeongbaekchul-san, and assessment of its antiadipogenic effect. Saudi Pharmacol. J. 2019, 27, 145-153. [CrossRef]

23. Seo, C.S.; Shin, H.K. Simultaneous determination of 12 marker components in Yeonkyopaedok-san using HPLC-PDA and LC-MS/MS. Appl. Sci. 2020, 10, 1713. [CrossRef]

24. Jung, M.Y.; Seo, C.S.; Baek, S.E.; Lee, J.; Shin, M.S.; Kang, K.S.; Lee, S.; Yoo, J.E. Analysis and identification of active compounds from Gami-Soyosan toxic to MCF-7 human breast adenocarcinoma cells. Biomolecules 2019, 9, 272. [CrossRef] [PubMed]

25. Seo, C.S.; Shin, H.K. Quality assessment of traditional herbal formula, Hyeonggaeyeongyo-tang through simultaneous determination of twenty marker components by HPLC-PDA and LC-MS/MS. Saudi Pharmacol. J. 2020, 28, 427-439. [CrossRef] [PubMed] 
26. International Conference on Harmonisation (ICH). Q2B, Validation of analytical procedures: Methodology. November 1996. Available online: https: / www.fda.gov/regulatory-information/search-fda-guidance-documents/q2b-validation-analyticalprocedures-methodology (accessed on 22 December 2020).

27. U.S. Food and Drug Administration. Reviewer Guidance, Validation of Chromatographic Methods; Center for Drug Evaluation and Research: Rockville, MD, USA, 1994. 